Manuelle Medizin 2008 · 46:380 DOI 10.1007/s00337-008-0658-6

(c) Springer Medizin Verlag 2008
Stefan Kopp

Zahnärztliches Universitätsinstitut der Stiftung Carolinum, Goethe-Universität Frankfurt am Main

\title{
Hier stehe ich, ich kann nicht anders, .....
}

Wenn es um die Funktion in der Zahnmedizin und der Kieferorthopädie geht, ist man geneigt, die letzten Worte des Reformators auf dem Reichstag zu Worms im Jahre 1521 auf den Lippen zu haben. Freilich ist diese oft zitierte Version wissenschaftlich nicht belegt. Im Original ist überliefert:, $[\mathrm{Da}$ ] ... mein Gewissen in den Worten Gottes gefangen ist, ich kann und will nichts widerrufen, weil es gefährlich und unmöglich ist, etwas gegen das Gewissen zu tun. Gott helfe mir. Amen."

Und genau darum geht es. Todgesagte leben länger - so sagt man. In den vergangenen Jahren hat man uns und das Thema Funktion in der Zahnheilkunde schon öfter einmal zu Grabe getragen. Verspottet und subsumiert unter dem Begriff „Gnathosophie" galten Zusammenhänge zwischen Okklusion und Schmerzen/Dysfunktionen im craniomandibulären System allenfalls als nicht gesicherte Hypothese.

Was Mitte der 8oer Jahre des vergangenen Jahrhunderts in Kiel zusammen mit Prof. Ewers und Prof. Buchmann unter gewebespezifischer Diagnostik und Therapie aus der Taufe gehoben wurde und durch die interdisziplinäre Zusammenarbeit mit Dr. Gernot Plato aus Rendsburg und Prof. Seifert aus Bad Segeberg von der Manuellen Medizin und der Hals- Nasen-, Ohrenheilkunde dankbar aufgenommen wurde, war in der Zahnmedizin nicht salonfähig. Erstaunlich, da der HNO-Arzt
Costen schon 1934 die Zusammenhänge kannte und detailliert beschrieb.

Aber es gibt glücklicherweise Kollegen, die jeden Tag noch Patienten behandeln "müssen“ - auch die wenig Geliebten mit Schmerzen und Dysfunktionen im Bewegungssystem, die man ja von anderer Seite so gerne und oft ausschließlich den Fächern Psychosomatik und Psychiatrie zuschreibt. So hat sich das Thema einer effektiven interdisziplinären Funktionstherapie unter elementarer Beteiligung der Zahnheilkunde bis heute gehalten. Unter der Präsidentschaft von Prof. Georg Meyer aus Greifswald, der - sachrichtig und orthographisch perfekt - den Deutschen Zahnärztetag 2005 in Berlin unter dem Begriff ZahnMEDIZIN zelebriert hat, wurde unser Anliegen wieder dort platziert, wo es auch hingehört: mitten in die interdisziplinäre Medizin.

Aus diesem Grunde ist es gut und sehr zu begrüßen, dass die Dezemberausgabe der Manuellen Medizin sich einmal mehr dem Thema „Interdisziplinäre Therapie von funktionsorientierten Beschwerden“" annimmt.

Evidence Based Medicine oder Experience Based Medicine? Ersteres wird als Forderung zur Verbesserung der diagnostischen und therapeutischen Ergebnisse gefordert. Das ist gut so. Leider liegen noch - relativ wenig vergleichende Studien zum wissenschaftlichen Beweis für die Effektivität diagnostischer und therapeutischer Verfahren gerade in der funktionsorientierten Therapie von Schmerzen und Dysfunktionen im Bewegungssystem vor. Mit dem vorliegenden Heft kommen wir aber einem von Vielen akzeptierten Untersuchungsstandard und einer klinisch praktikablen Therapieform - wie schon bei Ahlers et al. oder bei Ernst/Freesmeyer zu studieren - näher. Wir finden Vorschläge und Lösungsmöglichkeiten bis hin zur komplexen prothetischen Versorgung.

Dank an alle, die mitgearbeitet haben. Freude und Motivation allen, die das Niedergelegte gleichermaßen in der täglichen Praxis und der Wissenschaft umzusetzen gewillt sind.

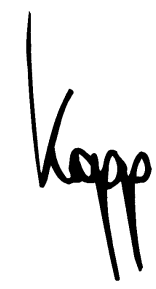

S. Kopp

\section{Korrespondenzadresse}

Prof. Dr. Stefan Kopp

Zahnärztliches Universitätsinstitut der Stiftung Carolinum

Goethe-Universität Frankfurt am Main

Theodor-Stern-Kai 7

60596 Frankfurt am Main

kopp@med.uni-frankfurt.de

\section{Mehr in Heft 1 / 2009}

Liebe Leserinnen, liebe Leser, um der Komplexität und dem Facettenreichtum der CMD gerecht zu werden, wird sich auch die erste Ausgabe 2009 noch einmal ausführlich der Thematik CMD widmen. 